\title{
Systems genetics links Bicc1 to bone development
}

A new study in mice and humans shows that $\mathrm{BMD}$, one of the strongest predictors of osteoporotic fractures, is positively regulated by protein bicaudal $\mathrm{C}$ homologue 1 (Bicc1), an RNA-binding protein involved in kidney development and implicated in polycystic kidney disease (PKD). "Therapeutically targeting Biccl has the potential to increase BMD, thereby decreasing an individual's risk of developing osteoporosis or sustaining an osteoporotic fracture," speculates Charles Farber, senior author of the study.

In their previous work, the researchers used linkage analysis to identify quantitative trait loci (QTLs) for BMD in a cross between mice with low bone mass and mice with high bone mass. In the new study, the team used a systems genetics approach to identify the gene responsible for a BMD-associated QTL on mouse chromosome 10. As Farber explains, "systems genetics studies seek to identify genetic variation that influences gene expression and then use this information to more rapidly identify the subset of variation that contributes to disease".

The researchers used microarray analysis to identify a subset of genes located on chromosome 10 whose transcript levels were influenced by local genetic variation and whose expression correlated with BMD. Nine genes fitting these criteria were identified, of which only Biccl was predicted by causality modelling to be responsible for the change in BMD. To validate this prediction in vivo, the researchers studied Bicc1deficient mice. As Bicc1 ${ }^{-/-}$mice develop severe early-onset PKD and die shortly after birth, the team used Biccl ${ }^{+/-}$mice. BMD was significantly lower in Biccl ${ }^{+/-}$ mice than in their wild-type littermates, confirming that Bicc1 is a genetic determinant of BMD.

To further investigate how Bicc1 influences BMD, the researchers used a co-expression network-based approach to identify connections between Bicc1 and other genes. Biccl (whose function in bone is not known) was shown to be highly co-expressed with a group of genes involved in osteoblast differentiation, most notably with polycystin-2 ( $P k d 2)$, which encodes a $\mathrm{Ca}^{2+}$-channel protein expressed in the kidney and osteoblasts. Knockdown of either Biccl or $P k d 2$ in primary calvarial osteoblasts impaired osteoblast differentiation. Furthermore, $P k d 2$ overexpression rescued defects in osteoblast function resulting from Bicc1 deficiency. Overall, the findings suggest that Bicc1 influences BMD by regulating osteoblastogenesis, most probably through transcriptional regulation of $P k d 2$.

The wider relevance of the findings to bone biology was demonstrated in two meta-analyses of human BMD genome-wide association data. Single nucleotide polymorphisms (SNPs) in both BICC1 and PKD2 were significantly associated with femoral neck BMD. A BICC1 SNP was also associated with lumbar spine BMD.

"This discovery increases our understanding of the genetic factors affecting BMD and highlights the power of systems genetics for complex trait gene discovery and functional characterization," conclude the authors in their report.

David Holmes

Original article Mesner, L. D. et al. Bicc1 is a genetic determinant of osteoblastogenesis and bone mineral density. J. Clin. Invest. doi:10.1172/JCI73072 